(O2021 IEEE. Personal use of this material is permitted. Permission from IEEE must be obtained for all other uses, in any current or future media, including reprinting/republishing this material for advertising or promotional purposes, creating new collective works, for resale or redistribution to servers or lists, or reuse of any copyrighted component of this work in other works. 


\title{
Integration of a Human-aware Risk-based Braking System into an Open-Field Mobile Robot
}

\author{
José C. Mayoral ${ }^{1}$ Lars Grimstad ${ }^{1}$ Pål J. From ${ }^{1}$ Grzegorz Cielniak ${ }^{2}$
}

\begin{abstract}
Safety integration components for robotic applications are a mandatory feature for any autonomous mobile application, including human avoidance behaviors. This paper proposes a novel parametrizable scene risk evaluator for openfield applications that use humans motion predictions and predefined hazard zones to estimate a braking factor. Parameters optimization uses simulated data. The evaluation is carried out by simulated and real-time scenarios, showing the impact of human predictions in favor of risk reductions on agricultural applications.
\end{abstract}

\section{INTRODUCTION}

Labor shortages and the need to increase food production have accelerated agricultural robotics' developments. However, safety surrounding the robots operating in agricultural environments is still an open problem that requires further effort to ensure safe and reliable operation in complex situations.

Generally, the safety systems for autonomous robots consider human detection and obstacle avoidance separately to reduce the problem's complexity. However, a complete solution to safety in agriculture must consider situation awareness, especially in applications where hazardous tools must perform a task.

Moreover, those systems must guarantee a complete safety system, which may meet a set of safety standards for risk assessment and risk reduction, including human awareness navigation which ensures that any human is not at risk in an environment where robots and humans must interact. These safe systems must constrain the vehicle's motion to reduce risk.

The international safety standard ISO-13489 [21] emphasizes the importance of machine safety functions' identification. Integrating safety into robotic applications is a mandatory feature for any autonomous mobile application to ensure human safety, being one of the main components. Also, it states that any machinery must apply protective measures as a risk reduction procedure for safety-related control functions. The integration or modification of a safetyrelated part of a control system improves the avoidance of the dangerous effect of a system component.

To certify safety in a robotic system, it should avoid dangerous situations occurring in the vicinity of the robot workspace and not entice any safety rule breach. Due to the world's complexity, a common way to represent the world at a robotic application is to entitle the system to create

1 Norwegian University of Life Sciences (NMBU) \{jmayoral, lars.grimstad, pal.johan.from @nmbu.no

${ }^{2}$ University of Lincoln (UoL) gcielniak@lincoln.ac.uk a local map with selected features, which provides a discrete representation of the environment [11]. Safety-related international standards like ISO-12100 [20], ISO-18497 [22] or ISO-13489 underline the need for risk calculation and reduction systems safety-related applications.

Therefore, an application-specific solution for safe autonomous tasks in agriculture must consider the above constraint, especially when an autonomous robot carries potentially hazardous tools, especially in applications that require power tools, like harvesting, seeders, or cultivators. An example, for grass harvesting application, a mobile robot brings a cutter, Figure 1 shows a Thorvald robotic platform [8] suited for this application where a cutter is attached to its frontal part.

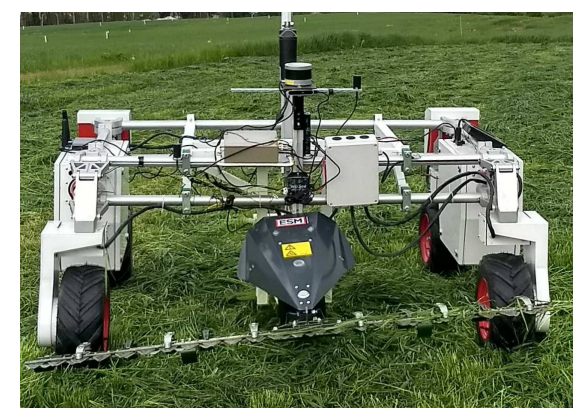

Fig. 1. Thorvald Platform suitable for grass cutting.

Inspired by the need for safety-reduction methods on autonomous tasks to achieve the standards, we propose a novel human-aware safety system for agricultural robots based on risk assessment. The specific contributions of the paper include:

- a parametrizable safety braking system based on local risk detection using sampled human motion prediction.

- experimental optimization and evaluation in a simulated scenario.

- validation of the optimized system parameters in a realworld scenario.

\section{Related WORK}

Safety by definition is abstract, and it adapts within the application, and an observer perception [16]. In applications that require power tools, i.e., agriculture, human avoidance techniques become insufficient due to the nature of the task and the person's risk.

Human safety became one of the main challenges [27, $24]$ in the context of autonomous mobile robots. Therefore, application-specific hazard analysis must trigger the safety 
systems development where any robot acts [27]. From the international standards' point of view, ISO-10218 [19] states a reduced speed control as a general requirement for safety.

An autonomous driving vehicle's safety system, and every subsystem, must meet high safety standards [1]. One method to get this in to focus on the human-robot collaboration [25] which limitation is that a mistaken human interaction can lead to failure, injuring the person.

Another approach bases on risk assessment, a risk-scoring method defined as a weighting sum of risk elements, gives a quantitative measurement of any potential risk [5]. Additionally, a static environmental risk map encloses a risk index based on the cell's probability of occupancy over time, applying a set of weighted risk elements according to the application. Further, Majumdar et al. [12] state cost and worse-case metrics as the most common risk metrics. Inspired by this, they develop an axiomatic risk theory, where six axioms must be satisfied to create feasible risk metrics for robotic applications.

\section{A. Costmap Layers}

Costmaps are the most common environmental representation used in autonomous applications [11]. According to Kruse [9], a temporary environment representation constructs like costmaps, clusters navigation cost functions. Those representations consist of several layers, where each one provides further information over the environment.

Layers can condense environmental information. Brouwer compares different information layers, including a behavioral grid that holds the cell's occupancy probability on close time, and fuses them into a probability map evaluating those models through evaluation parameters [2]. Additionally, it determines each pedestrian's collision probability based on their location relative to the car path.

On the other hand, environmental features can be integrated into costmaps, including terrain features as height [7], person semantics integration [28], and obstacle congestion [14]. After generating a costmap, a motion planner must process the environmental data to generate a near-optimal trajectory.

Sampling-based planners have succeeded in solving robot motion's problem [6], and those take into account the kinematics of the robot to execute the optimal trajectory. A collision checking or an avoidance algorithm finishes the motion planner pipeline to avoid unwanted situations.

\section{B. Human-aware Navigation}

In mobile robots, safe navigation and human perception share a connection that entails safety in an autonomous application. There exists a wide range of solutions for socialaware navigation designed to perform at scenarios where humans and robots share the motion space according to Chik's team [4]. They give some inputs on several navigation frameworks and state that the main disadvantage of predictivebased architectures is that the awareness functionalities trust the planner's performance.
Rudenko et al. [17] condense the human motion trajectory prediction approaches, showing an increased interest in pattern-based approaches over the physics-based approaches to exploit data for human trajectory prediction solutions.

In the scope of human-robot interaction(HRI), Lasota [10] split the definition of safe HRI into control, motion, prediction, and psychological considerations methods. In this classification, the prediction group collects human and robot activity and motion algorithms, covering raw data collection until predicting actions. A descriptive analysis of several short and long-term predictions introduces the implications and constraints required to perform a motion prediction, analyzing the assumptions and periodic horizon.

Trajectory prediction requires a data association procedure to match consecutive detections, assigning them a unique id. As a data association solution, a statistical motion prediction algorithm uses the clusters' mean and standard deviations to evaluate the distance between previously computed trajectories, adding the new matched element to the previously computed trajectories [26].

\section{Braking Systems}

In specific applications, like agriculture or driving, an emergency braking system must avoid a collision in scenarios where perception has a significant role in a safe navigation system. Autonomous Emergency Braking (AEB) provides an additional independent safety layer for autonomous cars. According to the obstacles ' positions, a partially observable Markov decision model braking approach estimates its braking states [3]. Similarly, time-to-brake and time-to-collision indicators can represent a safe evaluation metric [18].

Another solution is introduced on [15] that models a braking system where an average deceleration factor is estimated in the function of velocity drop and calculates the braking force in terms of the terrain slope.

\section{APPROACH}

In the context of risk estimation, the system must detect any humans and grant them a safe performance where they are not at risk when sharing an environment with a mobile device. Our approach defines a braking system based on the risk estimation of humans. Here, a braking system complements the risk estimations, composing a human-safetycentered approach. The Figure shows our approach's overall description, showing the four steps of our approach, based on [27].

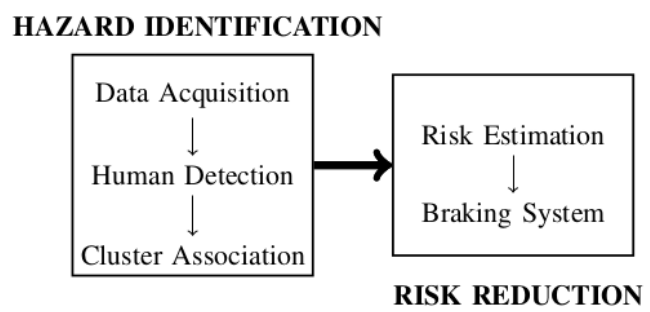

Fig. 2. Two-Stage Safety Estimation Pipeline. 
For this research, humans' estimation assumes that risk is a function of their poses and velocities and following possible motions. Further, a set of risk zones around the robot penalizes those motions. For instance, when a mobile agricultural robot executes tasks like harvesting on open-fields, it will likely move forward during most of the execution. Therefore, the higher risk zones should be in the robot's forward direction.

Furthermore, as mentioned by [10], prediction can act as a safety feature because it estimates how the scene will change on a temporal window, then, human velocity vectors indicate risk estimations.

\section{A. Hazard Identification}

Given human poses and speed vectors, risk zones definition, our approach estimates the risk resampling every person's current pose given its velocity vector. Our resampling method is a depth-limited search for a maximum depth D.

During the Hazard identification stage, the process starts with a generic 3D human detector, followed by a cluster association algorithm that matches consecutive human detections. Our approach assumes that the human detection's output is 3D points' clusters provided on the sensor coordinate system.

A comparison between previously associated detections and current detection is mandatory for updating detected humans states. Apriori comparing the queue $(\mathrm{Q})$ and current (C) detections, the last must be transformed into a world frame. This condition is required further for motion directions estimation. We propose a modified version of the association solution on [26] that exploits likelihood along the three-axis.

$A$ vector $F$ represents the mean values and standard deviation of the 3D axis (X, Y, Z). The mean absolute error along with all the queued elements and the current detected vector $f^{c}$ compute the term $b_{c}^{t}$ for each detection in $\mathrm{C}$.

$$
b_{c}^{t}=\min _{\forall q \in Q}\left(\sum_{f}^{F}\left|f^{q}-f^{c}\right|\right)
$$

If the likelihood $b_{c}^{t}$ is lesser than a matching threshold $m_{t h r}$ then, the queued element $\mathrm{s}$ is updated with the element c.

$$
Q_{s}^{t}= \begin{cases}\underset{\forall q \in Q}{\operatorname{argmin}}\left(\sum\left|f^{q}-f^{c}\right|\right), & \text { if }\left(b_{c}^{t} \leq m_{t h r}\right) \\ n e w_{o b j}, & \text { otherwise }\end{cases}
$$

The motion direction and velocity vector are two essential features for developing a safety framework. Both estimates update the cluster information after association.

Additionally, our approach includes the following features:

- Humans not matched after a time threshold are no longer consider in the queue.

- If two object trajectories intersect, our algorithm allows matching multiple new detections with the same queued detection.
After, our algorithm filters out the current humans in the scene, updating their states with the latest measurements. The risk can be estimated based on their current state.

\section{B. Risk Reductiion}

The risk estimation considers additional task-related and dynamic-based cues. This step requires additional specifictask information tasks defined as risk zones, provided as a grid map layer. For integrating dynamic-based features, a model-based approach uses each human state gotten as a result of the matching procedure.

The ISO26262 [23], defines severity as "the amount of damage or harm a hazard could create" for autonomous driving applications, where the highest classification means major consequences like death or significant system loss on scenarios in the scenarios where humans and robots cooperate.

Expecting that risk increases on a robot equipped with machinery to perform an agricultural task while detecting a human in the motion's direction and at the tool's orientation might have the highest risk. Consequently, there must be a risk difference between the proximity of the robot and a person as a function of humans and robot characteristics.

Therefore, detected persons in the proximity of any power machinery are under a higher risk than those on regions on the robot's sides. A visual explanation of such a situation in Figure 3 highlights the risk zones where the robot's front has a higher risk than a person detected behind the robot.

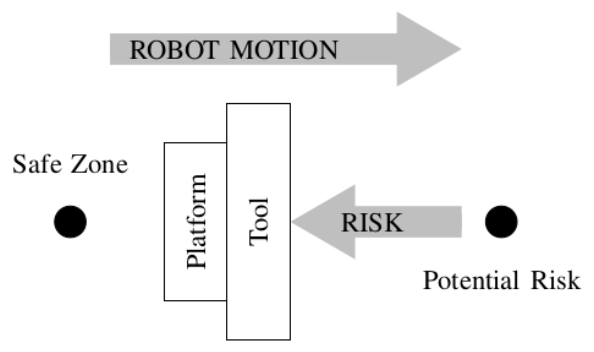

Fig. 3. Overlap between power tool and hazard level.

Toward the grid map layer representation, risk zones use geometric shapes projected on a grid layer $\mathrm{S}$ where all the cells insides a polygon shares the risk value [0 - 255], doing possible to create multiple risk level zones, penalizing objects moving towards the riskiest zones.

To deal with motion prediction complexity, we add a set of real-time layers $m^{t+d}$, one for each prediction timestep $d$ with a maximal depth D. Every layer condenses the motion predictions for the detected humans at time $t$.

As an online safety system, it calculates the layers with a sample-based trajectory prediction based on detected humans' current position assuming that the speed stays constant for a fixed time in the future.

Further, this can be used to recursively sample the object's plausible positions at the next time steps, similar to a classic motion planner, but applied to the detected humans themselves. Assuming a simple linear motion model. 


$$
X_{t+d+1}^{j}=\left(\begin{array}{c}
x_{t+d}^{j} \\
y_{t+d}^{j} \\
\theta_{t+d}^{j}
\end{array}\right)+\left(\frac{d \vec{x}_{t+d+1}^{j}}{d t} * \Delta_{t}^{*}\right)
$$

Where $\Delta_{t}^{*}$ is handset parameters that allow deciding the predicted layers' time steps and $\frac{d x_{t+d+1}^{*} \vec{d}^{s}}{d t}$ stands for the possible velocity after a motion primitive is applied.

Each person poses $\mathrm{X}$ with depth $\mathrm{d}$ has $\mathrm{J}$ possible motions defined according a set of motion primitives (move forward, turn right, and turn left and stay). Every motion primitive $\mathrm{j}$ for each time step d has a fix probability $P\left(X_{t+d+1}^{j} \mid u_{t}^{j}\right)$ of occurrence where:

$$
\sum_{j}^{J} P\left(X_{t+d+1}^{j} \mid u_{t}^{j}\right)=1
$$

Simultaneously, a depth factor with a decay factor $\mathrm{K}$ maximizes the impact of the motions sampling closer to the current over the risk estimation, as the lower the depth higher the impact.

$$
P\left(R_{t+d}\right) \propto R(t+d)=\exp ^{(-K * d)}
$$

The following equation merges each primitive motion and the depth factor's probability.

$$
P\left(R_{t+d} \cap X_{t+d+1}^{j} \mid u_{t}^{j}\right)=P\left(R_{t+d}\right) \times P\left(X_{t+d+1}^{j} \mid u_{t}^{j}\right)
$$

Given a known similarity transformation between the observation and a grid map frame coordinates $T_{m}^{c}$. The motion prediction layer $m^{t+d}$ is updated as described on the function. Note that each motion sample updates one single cell.

$$
P\left(m_{c x, c y}^{t+d}\right)=\max _{\forall j \in J}\left|P\left(m_{c x, c y}^{t+d}\right), P\left(R_{t+d} \cap X_{t+d+1}^{j}\right)\right|
$$

Up to this point, our approach creates a single layer for each recursive iteration in the depth-limited search sampling procedure, condensing several time instances $(t+d)$ calculated during t. A final layer clusters all d layers setting its values according to each cell's maximum value at all the d layers in time instance $\mathrm{t}$, creating a probability layer $\mathrm{L}$.

$$
P\left(L_{c x, c y}^{t}\right)=\max _{\forall d \in D}\left|P\left(m_{c x, c y}^{t+d}\right)\right|
$$

The timed risk map, defined on eq. 7 bases on the probability of risk over time. A final calculation for the scene risk probability at time $\mathrm{t}$ comes from the product between layer L times the safety region layer S.

$$
P(\text { risk })=\max \left|L^{t} \odot S^{*}\right|
$$

The risk estimations compute online due to run-time constraints and do not integrate previous risk estimations. Every new human detector data estimates an independent risk calculation. The braking system considers the higher human risk value to decelerate the autonomous platform to reduce the system's risk through a braking system proportionally to the risk (on equation 9).

$$
v_{t}^{\text {safe }}=v_{t} \times(1-P(\text { risk }))
$$

Our approach reduces risk, integrating a braking system according to the scene's human risk estimation. This approach works parallel to the navigation system, and it acts independently to the motion planner.

\section{Risk Zones Selection}

A point-cloud detector using a Velodyne LiDar provides the human 3D bounding boxes for our approach due to it provides a $360^{\circ}$ solution. The detection range is the primary component to define the risk zones. After a data record session, a map of the detection system's capabilities confers the spatial constraints, at Figure 4.

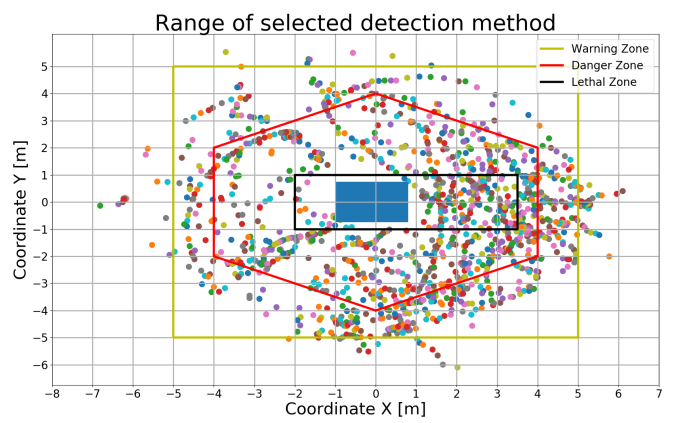

Fig. 4. Overlay of Human Detector Range and Custom Risk Zones.

For cases where the person is below the minimum distance range, a complimentary emergency braking system counters back the range sensor's range limitation setting a lethal zone, where if the sensor detects any point in that region, the robot must stop.

\section{Parameters Selection}

In the context of human-aware navigation for our braking system, the selection of parameters will be hard to find while recording data in the real world due to the complex human motion behavior in real life.

Our approach requires a set of parameters whose values impact the system's overall performance. Due to the nature of the task, optimizing those at real-world conditions might not be the safer idea, including the high number of samples required to get a robust solution.

Therefore, the optimization process prunes the parameter using a Gazebo simulated environment, consisting of a simulated robot and a human who moves randomly in the environment. In that simulation, the robot moves a fixed distance and comes back to the start point, and with each iteration, a random walk updates the parameter values, including the simulated human trajectory. The simulation description can be seen in Figure 5.

Those parameters include timestep $\Delta_{t}^{*}$, maximum depth $\mathrm{D}$, and a primitives' number $\mathrm{J}$. As evaluation criteria for 


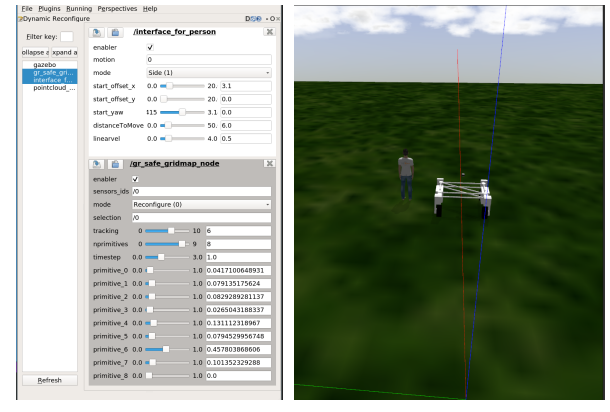

Fig. 5. Gazebo environment for a simulated robot and person, showing a set of re-configurable parameters.

parameters sets, and we select the security metrics defined on [13]. We group the iterations according to timestep, D, and $\mathrm{J}$ parameters for selecting the best parameters set and select the best worst-case performance. This process assumes that every $P\left(X_{t+d+1}^{j} \mid u_{t}^{j}\right)$ probability is proportional to its impact on the risk score.

Over ten thousand executions in our simulator, the best parameter set is shown on table I. As a remark, our method finds the most optimal parameter set on using just five primitives, avoiding backward sampling primitives.

TABLE I

BEST PARAMETERS SET.

\begin{tabular}{|c|c|c|c|}
\hline Name & Description & Symbol & Value \\
\hline Timestep & - & $\mathrm{dt}$ & 0.1 \\
\hline Track. & - & $\mathrm{D}$ & 4 \\
\hline \#Prim & - & $\mathrm{J}$ & 5 \\
\hline Prob. $P_{0}$ & Forward & $P\left(X_{t+d+1}^{0} \mid u_{t}^{0}\right)$ & 0.247 \\
\hline Prob. $P_{1}$ & Forw. Right Turn & $P\left(X_{t+d+1}^{1} \mid u_{t}^{1}\right)$ & 0.273 \\
\hline Prob. $P_{2}$ & Forw. Left Turn & $P\left(X_{t+d+1}^{2} \mid u_{t}^{2}\right)$ & 0.273 \\
\hline Prob. $P_{3}$ & Backw. Right Turn & $P\left(X_{t+d+1}^{3} \mid u_{t}^{3}\right)$ & 0.102 \\
\hline Prob. $P_{4}$ & Backw. Left Turn & $P\left(X_{t+d+1}^{4} \mid u_{t}^{4}\right)$ & 0.102 \\
\hline
\end{tabular}

\section{EXPERIMENTS}

Human motion in outdoor scenarios as open-fields encores a complex problem, especially when measuring safety. Therefore, the experiment evaluates our method with the optimal parameters set using SM1 and SM2 metrics.

\section{A. Experimental Design}

For the experimental design, Thorvald [8] is chosen as the platform, equipped with a Velodyne VLP-16 sensor. Our validation method focuses on the braking system's performance at a set of generic situations found on openfield scenarios while the robot moves forward and returns a distance of 10 meters while a single person is performing one of the next actions:

1) Stands close to the end position.

2) Walks towards the end pose of the robot.

3) Cross coming from any sides of the robot.

4) Random walks on the robot's surroundings.

Each test case runs several times, highlighting that humans have no constraints or predefined paths to follow, providing diverse paths. Each experiment calculates risk elements as statistical features of each test case in simulation and realworld performance. Our risk elements are inspired by the ones on $[12,13]$ :

TABLE II

RiSK ELEMENTS SELECTION.

\begin{tabular}{|c|c|}
\hline Description & ID \\
\hline Detected Distances Average & M1 \\
\hline Detected Distances Minimum (Worst Case) & M2 \\
\hline Detected Distances Maximum (Best Case) & M3 \\
\hline Detected Distances Variance & M4 \\
\hline Risk Average & M5 \\
\hline Risk Variance & M6 \\
\hline Distance-Risk Covariance & M7 \\
\hline
\end{tabular}

For the evaluation, we recall the risk estimation formula from [5] which uses all elements to calculate the robotic application risk. For this purpose, we consider each element as equally important. This assumption has the sole intention of evaluating the risk for each test case, either for simulation or real-world data.

\section{RESULts}

The relation between detected humans and risk zones in the context of risk estimation reveals consistency between estimated risk and risk zones, in figure 6. Black dots represent the human with a risk estimation value equal to a unit, while white dots mean no risk. Greyscale stands for persons who are not at lethal risk.

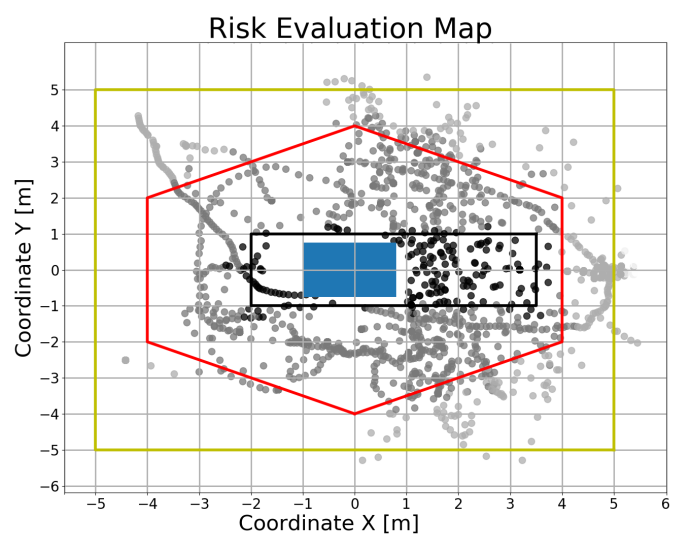

Fig. 6. Overlay of detected human and evaluated risk. Darker color recalls high risk.

The calculated risk elements are summarized on Table III. The Table shows each test case's features and RealWorld(R) and Simulation(S) scenarios. The maximal person detection distance (M3) is higher in simulated scenarios. As a consequence of simulated sensor characteristics, the perception system increases its detection range. Further, the risk average (M5) and its variance (M6) are lower in the simulated scenarios. Our data's covariance (M7) is negative in all experiments as expected, and a lower risk level means that the person is further. 
TABLE III

RISK ELEMENTS AND ESTIMATION RESUlTS.

\begin{tabular}{|c|c|c|c|c|c|c|c|}
\hline Case & M1 & M2 & M3 & M4 & M5 & M6 & M7 \\
\hline 1-Real & 5.03 & 3.78 & 7.12 & 0.36 & 0.46 & $\mathbf{0 . 3 2}$ & -0.29 \\
\hline 1-Sim & $\mathbf{6 . 1 4}$ & $\mathbf{5 . 1 3}$ & 6.97 & 0.22 & 0.01 & 0.00 & $\mathbf{- 0 . 0 1}$ \\
\hline 2-Real & 5.30 & 3.18 & 6.67 & 0.45 & 0.21 & 0.15 & -0.22 \\
\hline 2-Sim & 5.56 & 1.92 & 7.46 & 1.40 & 0.17 & 0.09 & -0.32 \\
\hline 3-Real & 3.34 & 1.02 & 6.22 & 1.25 & 0.80 & 0.18 & -0.28 \\
\hline 3-Sim & 4.76 & 1.55 & 8.23 & 1.34 & 0.43 & 0.07 & -0.24 \\
\hline 4-Real & 2.91 & 1.00 & 6.12 & 1.03 & $\mathbf{0 . 8 7}$ & 0.16 & -0.22 \\
\hline 4-Sim & 4.52 & 1.49 & $\mathbf{8 . 4 1}$ & $\mathbf{1 . 9 1}$ & 0.39 & 0.08 & -0.33 \\
\hline
\end{tabular}

The risk score method defined in [5] with balanced weights is selected to estimate the risk per scenario, and its scores are in the Table IV, as well as the number of samples per test case. Estimated risks at test case four, random motion, is the one that presents the maximum difference between environments, likely as a result of the motions' randomness.

TABLE IV

FinAL Risk SCORE PER TEST CASE

\begin{tabular}{|c|c|c|}
\hline Case & \# Samples & Risk Score \\
\hline 1-Real & 432 & $\mathbf{2 . 0 9}$ \\
\hline 1-Sim & 93 & 2.31 \\
\hline 3-Real & 125 & 2.03 \\
\hline 2-Sim & 182 & $\mathbf{1 . 9 7}$ \\
\hline 3-Real & 4097 & 2.01 \\
\hline 3-Sim & 1268 & $\mathbf{1 . 5 7}$ \\
\hline 4-Real & 2415 & 2.06 \\
\hline 4-Sim & 1063 & $\mathbf{1 . 4 9}$ \\
\hline
\end{tabular}

The minimal risk score is on the fourth scenario on a simulation environment that is the safer test case, mainly to the motion's randomness. In test case 1, fewer persons are detected because the person waits for the robot in a meeting point and, due to the absence of motion, the timebase sampling does not contribute to the risk estimation. The risk scores also show that the selected risk elements demonstrate that it is riskier to stands close to the goal pose. Since person velocity is zero, the re-sampling method does not provide any risk redundancy.

The first two cases contain fewer samples than the last two because they have a more extended interaction between the human a robot. The persons are longer in the detection range. Similar behavior appears in figure 6 close to the boundaries between lethal and danger zones. Also, it represents the impact of the risk evaluation's custom-designed risk zones.

The results show different performances between datasets, likely due to the risk element selection, the not-optimal weight selection for scoring, main weights for M2 and M3, or differences between real-world and simulated sensors. Simulation data reduces the risk of real-world experiments by simulating motions, in which a random walk selects parameter sets.

\section{CONCLUSIONS}

An approach to enhance a robot with a full-range braking system using simulations to prune the risk scoring procedure is this research's intention. Our method provides a practical quantitative people's risk assessment using a simple trajectory sampling and a predefined kinematic model to estimate risk over next time samples for every detected person. Simulated data allows our efforts to find safer parameters to evaluate risk in real-world scenarios.

Although this approach quantifies a person's risk based on static risk zones and samples of the detected person's feasible motions, unfortunately, it is still limited since it does not store information on the previous motions. The primitive motions' probabilities are static, and they do not update over time. As future work, additional safety metrics should complement the presented risk calculations. Also, multiple sensors will enhance the robot safety system's detection capabilities.

The approach provides solely a control strategy designed as a braking system. However, we suggest this method as the first step towards a more complex risk analysis on applications like agriculture. Any robotic system should evaluate the closer time-wise feasible scenarios to reduce risk on the autonomous task execution. This estimation should be considered a reference, not as a ground truth value. Further work must ensure the validity of the risk values.

\section{REFERENCES}

[1] Maria Binfet-kull, Peter Heitmann, and Christian Ameling. "System Safety for an Autonomous Driving Vehicle". In: International Connference on Intelligent Vehicles (1998), pp. 469-474.

[2] Nina Brouwer, Horst Kloeden, and Christoph Stiller. "Comparison and evaluation of pedestrian motion models for vehicle safety systems". In: IEEE Conference on Intelligent Transportation Systems, Proceedings, ITSC (2016), pp. 2207-2212.

[3] H. Chae et al. "Autonomous braking system via deep reinforcement learning". In: 2017 IEEE 20th International Conference on Intelligent Transportation Systems (ITSC). 2017, pp. 1-6.

[4] S. F. Chik et al. "A review of social-aware navigation frameworks for service robot in dynamic human environments". In: Journal of Telecommunication, Electronic and Computer Engineering 8.11 (2016), pp. 4150.

[5] Rob Dupre and Vasileios Argyriou. "Risk Estimation in Robotics and the Impact of Human Behaviour". In: Legal, Ethical and Socioeconomic Impacts, George Dekoulis, IntechOpen, Dec. 2017.

[6] M Elbanhawi and M Simic. "Sampling-Based Robot Motion Planning: A Review". In: IEEE Access 2 (2014), pp. 56-77. ISSN: 2169-3536.

[7] Péter Fankhauser and Marco Hutter. "A Universal Grid Map Library: Implementation and Use Case for Rough Terrain Navigation”. In: Robot Operating System (ROS) - The Complete Reference (Volume 1). Ed. by Anis Koubaa. Springer, 2016. Chap. 5. 
[8] Lars Grimstad et al. "On the design of a low-cost, light-weight, and highly versatile agricultural robot". In: Proceedings of IEEE Workshop on Advanced Robotics and its Social Impacts, ARSO 2016-March (2016).

[9] Thibault Kruse et al. "Human-aware robot navigation: A survey". In: Robotics and Autonomous Systems 61.12 (2013), pp. 1726-1743.

[10] Przemyslaw A. Lasota, Terrence Fong, and Julie A. Shah. "A Survey of Methods for Safe Human-Robot Interaction". In: Foundations and Trends in Robotics 5.3 (2017), pp. 261-349.

[11] David V. Lu, Dave Hershberger, and William D. Smart. "Layered costmaps for context-sensitive navigation". In: IEEE International Conference on Intelligent Robots and Systems (2014), pp. 709-715. ISSN: 21530866.

[12] Anirudha Majumdar and Marco Pavone. "How Should a Robot Assess Risk? Towards an Axiomatic Theory of Risk in Robotics". In: Robotics Research. Ed. by Nancy M. Amato et al. Cham: Springer International Publishing, 2020, pp. 75-84.

[13] Nelson David Munoz Ceballos, Jaime Alejandro, and Nelson Londono. "Quantitative Performance Metrics for Mobile Robots Navigation”. In: Mobile Robots Navigation May 2014 (2010).

[14] Alyssa Pierson et al. "Dynamic risk density for autonomous navigation in cluttered environments without object detection". In: Proceedings - IEEE International Conference on Robotics and Automation 2019May (2019), pp. 5807-5814. ISSN: 10504729.

[15] Mihail Pivtoraiko, Alonzo Kelly, and Peter Rander. "Efficient Braking Model for Off-Road Mobile Robots". In: Field and Service Robotics. Ed. by Peter Corke and Salah Sukkariah. Berlin, Heidelberg: Springer Berlin Heidelberg, 2006, pp. 541-552.

[16] Andreas Reschka. "Safety Concept for Autonomous Vehicles”. In: Autonomous Driving: Technical, Legal and Social Aspects. 2016. Chap. 23, pp. 474-476.

[17] Andrey Rudenko et al. "Human motion trajectory prediction: a survey". In: The International Journal of Robotics Research 39.8 (2020), 895-935.

[18] Markus Schratter et al. Pedestrian Collision Avoidance System for Scenarios with Occlusions. 2019. arXiv: 1904.11566 [CS.RO].
[19] International Organization for Standardization. ISO10218 Robots and robotic devices - Safety requirements for industrial robots - Part 1: Robots. Standard. Geneva, CH: International Organization for Standardization, 2018.

[20] International Organization for Standardization. ISO12100 Robots and robotic devices - Safety requirements for industrial robots - Part 1: Robots. Standard. International Organization for Standardization, 2013.

[21] International Organization for Standardization. ISO13489 Safety of Machinery - Safety-related parts of control systems - Part 1: General principles of design. Standard. International Organization for Standardization, 2015.

[22] International Organization for Standardization. ISO18497 Agricultural machinery and tractors - Safety of highly automated agricultural machines - Principles for design. Standard. Geneva, $\mathrm{CH}$ : International Organization for Standardization, Nov. 2018.

[23] International Organization for Standardization. ISO26262 Road vehicles - Functional safety. Standard. Geneva, CH: International Organization for Standardization, 2018.

[24] Torben Stolte et al. "Hazard analysis and risk assessment for an automated unmanned protective vehicle". In: IEEE Intelligent Vehicles Symposium, Proceedings June 2018 (2017), pp. 1848-1855. arXiv: 1704 . 06140.

[25] M. Vasic and A. Billard. "Safety issues in humanrobot interactions". In: 2013 IEEE International Conference on Robotics and Automation. 2013, pp. 197204.

[26] Dizan Vasquez and Thierry Fraichard. "Motion prediction for moving objects: A statistical approach". In: Proceedings - IEEE International Conference on Robotics and Automation 2004.4 (2004), pp. 39313936.

[27] Chinniah Y. "Robot Safety: Overview of Risk Assessment and Reduction". In: Advances in Robotics \& Automation 05.01 (2016), pp. 1-5.

[28] Cheng Zhao, Weixing Mei, and Wei Pan. "Building a grid-semantic map for the navigation of service robots through human-robot interaction". In: Digital Communications and Networks 1.4 (2015), pp. 253-266. 